\title{
Tehran traffic congestion charging management: a success story
}

\author{
H. Behruz, A. Safaie \& A. P. Chavoshy \\ Tehran Traffic Control Company, Tehran, Iran
}

\begin{abstract}
Tehran, the capital of Iran, is the largest city of the country with more than 8 million residents within an area of 600 square kilometers; it has more than 4 million vehicles and 4 million motorcycles as well, over a large scattered road network consisting of 4 active metro ways and 5 active bus rapid transit lanes. Nevertheless, deficiency of transportation systems in Tehran has led us to achieve the first place in the world in air and noise pollution.

At 1980, Tehran municipality lined a border around the city center, and defined a traffic congested area through which any entry for vehicles since then needs permission. Although such permission is only granted to a very small group of people, like physicians, there was still a great trespassing rate due to the insufficient capability of control. Until the past year, all the entry roads to the restricted zone were controlled by police officers.

There are as many as 5 million vehicles passing the borders each month of which $20 \%$ or 1.1 million are illegal. Thus, efficient control is a major need, and if systemized, it can deliver vital information on traffic data. In the past year, Tehran Traffic Control Company implemented an automated control of the borders, using 103 high resolution cameras which contributed to the detection of more than $80 \%$ of illegal entries, and has become a very useful tool to collect traffic data for further analysis. This paper discusses this success story.

Keywords: Tehran traffic problems, congestion charging, congestion pricing.
\end{abstract}

\section{Introduction}

Tehran is the capital and largest city of Iran with a population of over 12 million and an approximate city area of 600 square kilometers which, according to 
statistics, makes Tehran the sixteenth city in the world in terms of population density. Tehran also hosts nearly half of the country's industrial activity [1-4].

\subsection{Transportation and traffic in Tehran}

The city of Tehran includes an irregular road network, $2700 \mathrm{~km}$ in length, of which $14 \%$ are highways and $27 \%$ are arterial roads. Every day there are 15 million trips on the network with an average speed of $21 \mathrm{~km} /$ hour per vehicle (public and private), and the percentage of private vs. public vehicles (such as taxis) is estimated at respectively $52 \%$ and $58 \%$ [5].

Tehran is considered to be one of the most polluted cities in the world in terms of air and noise pollution. The worsening of this situation and the growing traffic congestion in public ways in recent years has led to a search for new ideas and measures to reduce the adverse effects of the traffic congestion. The most important of these measures was the limitation of vehicle traffic to the downtown area starting in 1980 and implemented in such a way that only a limited number of vehicles such as emergency vehicles can access the area.

Over time and as larger areas became afflicted by traffic congestion, the perimeter of the prohibited zone was increased, so that on weekdays only half of the city's vehicles can access these areas based on whether the last digit of the vehicle's license plate is odd or even. Figure 1 shows these two areas.

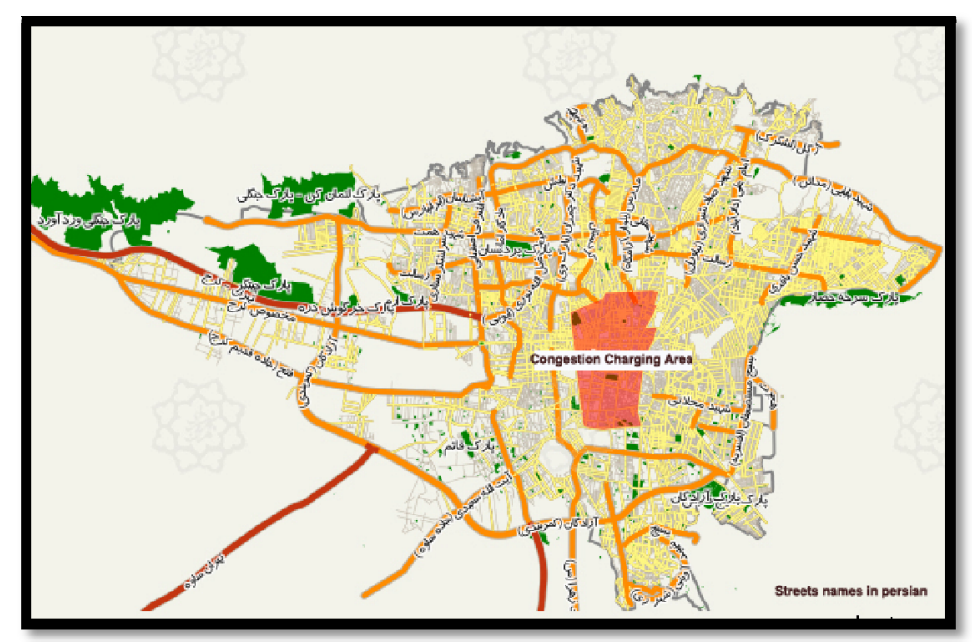

Figure 1: $\quad$ Limited areas where traffic is prohibited [3].

\section{Definition of the problem}

Many reasons for the traffic congestion and the high levels of air and noise pollution can be found in Tehran. Nearly 4 million cars and over 4 million motorcycles are driven in Tehran [6]. More than 400,000 cars in use are over 25 years old and more than 1.5 million vehicles are over 7 years old. Although a 
program to replace deficient vehicles has existed for many years, nonetheless during this same period the number of old vehicles (based on the informal definition given above) has continued to rise [7]. There are 2.5 million vehicles in service that have not received a certificate of technical examination. Newer vehicles also introduce high levels of polluting agents into the air due to lack of maintenance and due to the fact that the vehicles that are produced nationally are of lower quality.

Also, inadequate development of public transportation has led to unnecessary trips and has substantially increased the number of daily trips made by personal vehicles.

Geographically, Tehran is surrounded on three sides by mountains that slow the circulation of air, causing the air pollution to remain stagnant over the city. From a social point of view, Tehran is one of the densest cities in the world. This density is the main cause for innumerable trips within the city. Thus Tehran is one of the top countries in the world for air and noise pollution (see Figures 2 and 3 ).

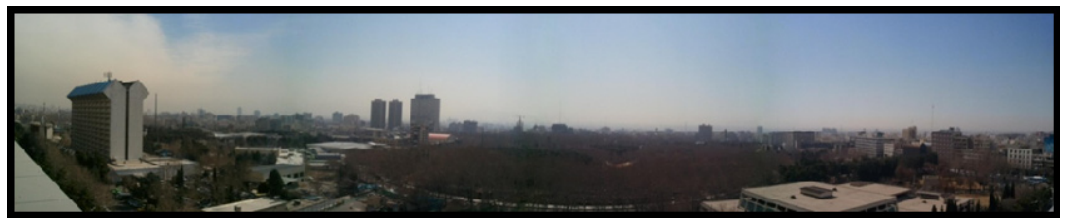

Figure 2: $\quad$ Air pollution in Tehran on a winter day.

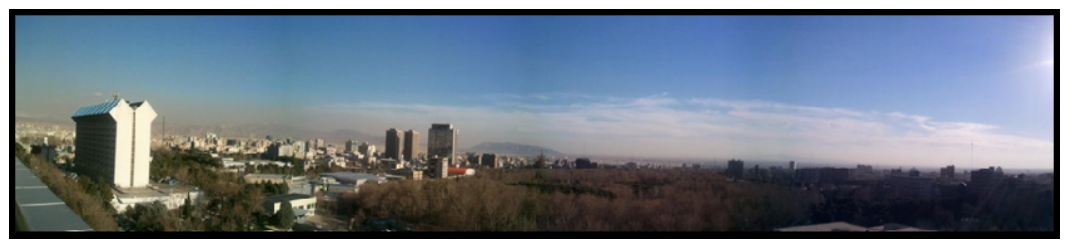

Figure 3: $\quad$ Reduced air pollution on a windy day, the day after, same time.

\section{History of the approach and procedures}

Starting in 1979 the limited downtown traffic area was designated in order to reduce air pollution and traffic congestion. It took the shape of an irregular quadrilateral. The area is open only to relief and emergency vehicles and certain groups including doctors and journalists during specific hours of the day. Since then, conditions of access to the area (hours of the day when restrictions apply, and the permit issuance system) have undergone some changes. Currently the project is implemented in two major traffic areas: the main project in the core area and the odd-even project in the outer layer area. When pollution conditions 
are extreme or at other critical periods, a combination of both the main traffic project and the odd-even traffic project is implemented.

Permits are issued each year providing access to the limited traffic zone. Upon payment of the established fees, drivers can use these permits. Recently there have been measures to create weekly and daily permits that will allow individuals to have access on a particular day or week of the year. The area covered by the plan is limited to downtown Tehran, consisting mainly of businesses and offices. Despite the restriction of traffic in this area, air pollution continues to be a major concern. One reason for this is the change in the pattern of pollution creation in the city. Over time, many city roads have become prone to congestion at different times of the day. In addition to the downtown area, many other pollution production centers have been created. Also, up until last year, the control of traffic entering the restricted area was achieved by human power, using police officers who, due to practical constraints, were able to prevent only about $20 \%$ of those entering the restricted area. This led the authorities to take a first step towards improving control over unauthorized entry to the restricted area by using the Intelligent Transportation System (ITS).

\section{The need for an effective control method}

The quadrilateral prohibited traffic zone crosses 103 roadways that are considered entrances to the prohibited traffic zone. Controlling these streets with human power would require at least 103 police officers who will enforce the access rules over the 10.5 hours during which the restrictions are effective. Considering the need for additional shifts due to high levels of traffic and also the need for additional police support in heavy traffic streets or highways this number can be estimated as 200. It should be noted that violations such as unauthorized entry into the prohibited traffic zone are of a completely different nature than other traffic violations; therefore traffic police training is also necessary and important. In addition to all of these requirements, human error is inevitable in systems that rely on human power and during periods of high activity the number of mistakes increases (in only one day, the total amount of traffic entering the prohibited traffic area consists of 230,000 cars $20 \%$ of which or 50,000 vehicles enter the area without permission).

Compared to systems based on computer processing, human powered systems have other weaknesses: there is no way to capture the data required for statistical analysis; the optimal performance of the police force requires a specially defined control mechanism; individual judgments when faced with delinquent drivers influence the enforcement of the law; human agents are affected by the climate; during police emergencies (such as special events) and when the staff is reduced, controlling some of the entry ways to the prohibited zone becomes difficult, and when several unauthorized vehicles enter the prohibited area at the same time, the number of undetected unauthorized vehicles increases. These factors led to the decision to use control mechanisms based on a video surveillance system in 2009. 


\section{The methodology for the implementation of automated control}

In 2007, a professional traffic control company (a subsidiary of the municipality of Tehran) conducted the basic studies and formulated the requirements necessary to implement automated controls. The first step was to review similar experiences in other cities around the world and the next step was to identify and select a system that would detect vehicles that enter the prohibited traffic zone.

\subsection{Similar experiences in other cities around the world}

Many cities around the world have started to deal with traffic congestion. For example, one can mention the implementation of the PROGRESS Project in European Union and traffic controls in London, Stockholm, Singapore, New York, New Jersey, California, Melbourne, Toronto and other localities. It is noteworthy that their approach to traffic control differs depending on their objectives, and can be either the control of an area such as in Tehran, or the control of some public ways such as in Singapore.

The experiences of other countries have been omitted in the interests of brevity and the fact that is readily available through internet.

\section{Available technologies}

Major components of traffic control systems are comprised of a sub-system to collect and record data (which would be capable of distinguishing unauthorized vehicles from the others), another sub-system to transfer the recorded data to the data center, and a sub-system to process the information which will also analyze and finalize the results obtained by the information gathering system.

\subsection{Data collection sub-system}

In general, information is gathered using one of two main methods: Many systems are based on sending and receiving waves (such as IR), while other systems record images of vehicles and then process the images for authentication purposes (focusing particularly on the license plate).

\subsubsection{Automatic number plate detection (ANPR)}

With this technology the vehicle's ID is the vehicle's plate and thanks to cameras equipped with ANPR systems installed in public ways, pictures of the vehicle will be taken. Then the technology selects the most appropriate image and uses an OCR (13) software to detect and extract the plate number. In some cases a vertical or horizontal displacement of only slightly more than $1 \%$ or $2 \%$ can reduce the system's efficiency and a change in target can cause fluctuations in the plate reading accuracy of $30 \%$ to $100 \%$ [8].

\subsubsection{DSRC (Dedicated Short Range Communications) technology}

In this system the ID of the vehicle is OBU (On Board unit) and is read by the Transceiver (installed on the antenna mast). One nice feature of this technology 
is that it can detect the direction of a moving vehicle with $100 \%$ accuracy, and this feature becomes very important when the fee is applied only to traffic moving in one direction [9].

\section{Automated control system in Tehran}

Based on the preliminary evaluation of transportation specifications in Tehran, ANPR was initially selected. In January 2007 two of the entry points to the Tehran prohibited traffic area were chosen and were equipped with cameras that recorded plate numbers. The implementation of this pilot project can be considered the starting point of all activities related to the automated control of Tehran's prohibited congested area.

\subsection{The pilot project}

The image capture was done in two selected intersections over 12 days and in three time periods between the hours of $8 \mathrm{am}$ to $9 \mathrm{am}, 1 \mathrm{pm}$ to $2 \mathrm{pm}$ and $5 \mathrm{pm}$ to $6 \mathrm{pm}$. The goal was to observe different hours of the day with different levels of natural light, various atmospheric conditions and taking into account the traffic load, during a 12-day period so the system's functions could be clearly seen. The analysis of the results of this field evaluation was as follows:

- $85 \%$ of passing vehicles were identified. The percentage of ANPR accuracy for the total number of passing vehicles was $75 \%$ and the total percentage of identified vehicles was $95 \%$.

- In high-speed streets the error rate of the system increases because of stretched images.

- During periods of high traffic congestion when the distance between cars is reduced it is possible that plates are hidden and cannot be read.

- Physical resemblance of Persian letters used in license plates, will be a problem for distinguishing the letters. Evidently letters such as "Sat" and "Sin" (two Persian letters that look physically similar) cannot easily be distinguished by OCR software.

- The installation positions in the streets that are points of entry to the prohibited traffic area require customized solutions to improve the quantity and accuracy of plates' identification. For instance, it may be necessary to install additional lighting.

- Dirty, damaged, bent, discolored plates, and those with nonstandard installation, or those with screws that are screwed on plates' numbers, or on occasions when a horizontal or vertical part of the picture of the plate is outside the view of the camera which makes the viewing and detection of the missing segment impossible, can in some cases negate the value of record collection.

\subsubsection{Identifying the challenges}

One of the most important challenges is the lack of legal infrastructure to implement and enforce this system over the long term. In order to penalize drivers, merely citing the video documents requires the existence of the 
necessary law in the country's civil code, although this issue can be solved in the short term by using the powers of the police.

If a technology other than ANPR technology is required, there will be major concerns over public acceptance, because drivers would be required to install the necessary equipment in their cars. This may lead to many forms of public rejection. In general, the broad deployment of this kind of equipment gives drivers a feeling of being controlled.

In addition, research shows that when drivers approach surveillance cameras, or any type of tracking device that is located on the road side, they unconsciously turn the steering wheel away from the camera. These unconscious movements which may lead to sudden lane changes or speed decreases, or deliberate movements such as driving very close to the vehicle in front at an unsafe distance so that the camera is unable to detect the license plate, actions which typically lead to dangerous accidents [10].

Improper modifications of the plate or the installation of the plate in an improper place that makes it impossible to be read, are among the deliberate and planned behaviors that drivers may use to undermine the system. Also, the existence of other objects that include numbers similar to the license plate placed in front or at the back of the vehicle can confuse the system and thus make the accurate detection of the plate's number impossible [10].

\subsubsection{Selecting the technology}

A comparison of the technologies in the categories of ANPR, DSRC and GPSGSM was made based on selection criteria. Using a form of multiple criteria analysis, and a rating system from 1 to 5 (very poor to very good), and taking into account the relative importance of each criteria to the rest, the scores were calculated for each given technology. The results show that the ANPR technology scored highly and would be the most appropriate technology to use within an automated control scheme if it is chosen for Tehran Traffic.

The scoring function for the technology is presented in the following equation:

$$
P t=\sum_{j=1}^{12} \sum_{i=1}^{5} w j(0.2 \times i \times C i)
$$

The first sigma in eqn. (1) is related to the standard row and the result will determine the standard weight of the technology. The second sigma in the above formula adds the results, which are the weight of different standards, from the first sigma together. $\mathrm{Wj}$ is the total weight of the standard, $\mathrm{i}$ is a number between 0 to $5, \mathrm{Ci}$ is a value equal to zero or one and the number 0.2 shows a progressive increase.

\subsubsection{Diagramming the operational workflow}

Figure 4 shows the operational workflow of the mechanized control system. The process begins by registering a high resolution color image of the vehicle and a black and white picture of the license plate and ends with issuing the violator a fine. 


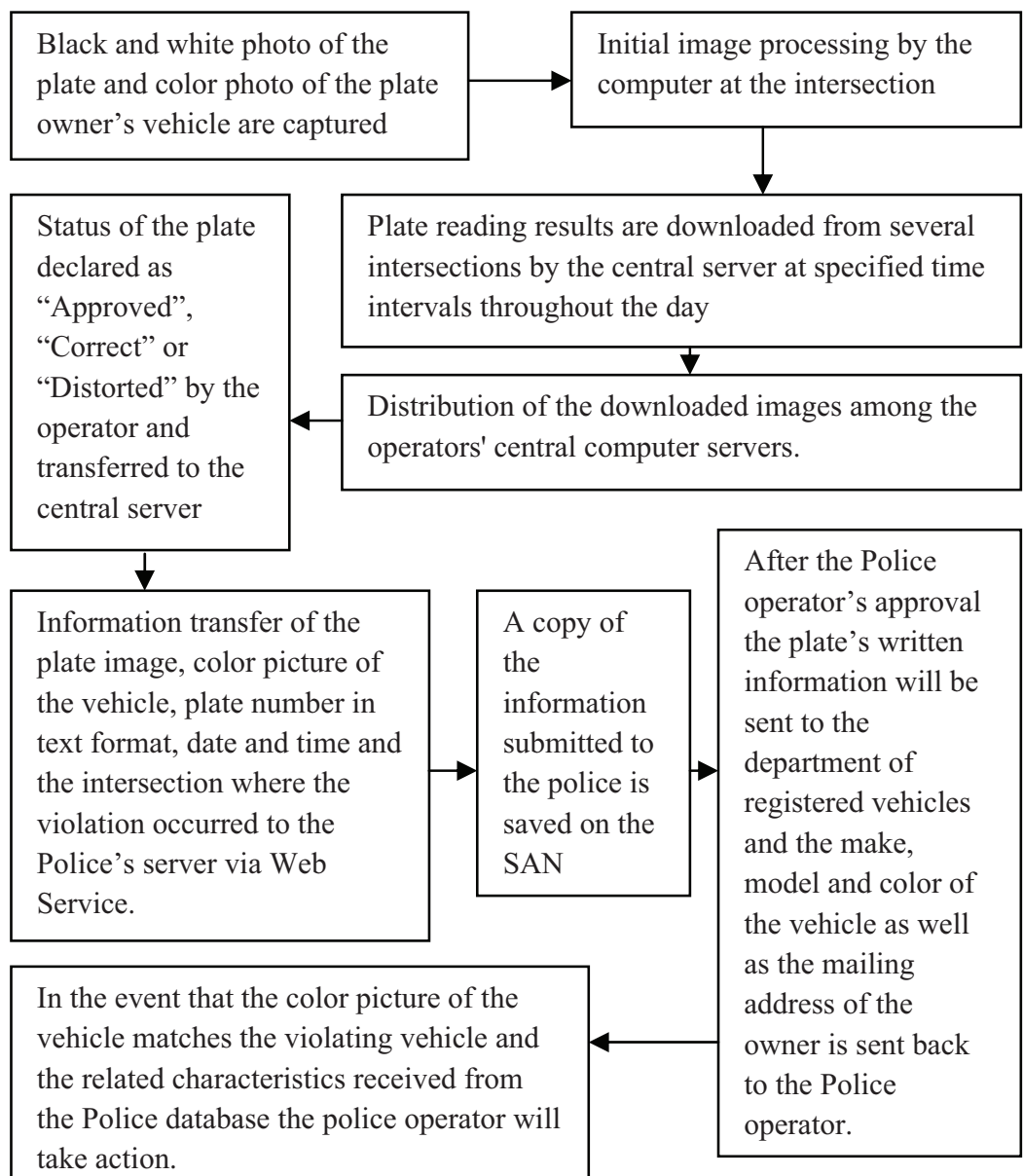

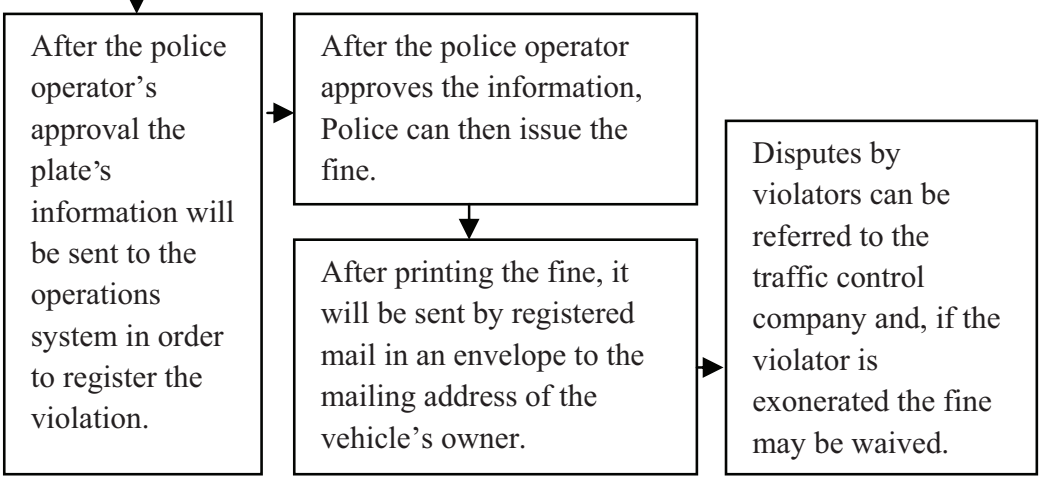

Figure 4: The operational workflow of the automated control system of the traffic plan in Tehran. 


\subsubsection{Important dates and project costs}

Project operations that included the purchase of cameras and equipment and the completion of the fiber optic communications platform began in April 2009 and ended in March 2010. Four months later, the official operation of this project began while the overall cost had been estimated at $\$ 10$ million.

\subsubsection{System's hardware specifications}

The quadrilateral prohibited traffic area includes 104 points of entry, some with two or three lanes. Consequently, more than one camera is often required to cover such intersections and so a total of 303 cameras were necessary to cover all intersections. Two separate teams are responsible for the implementation of the project and have used two major camera brands: Basler Products (BASLER vision technologies), and Prosilica (Allied Vision Technologies).The communications platform is used for the network communication of fiber optics, but some equipment is linked via wireless systems.

The system also includes a well-equipped center that supports its development and is responsible for fixing potential problems and failures.

An additional center has been embedded in the system, where several operators have the task of correcting the information that the ANPR system has read incorrectly from the plates (5\% of the total information). After this correction the system will read the plate again and will compare it to a list of authorized vehicles. Finally, the police will issue the fine without further involvement by the municipality.

\subsubsection{System's software specifications}

In order to view the surveillance camera images the web-based Omnicast software by Genetec is used. The plate reading algorithm operates in two steps: First every frame is searched to find the plate; next a search to identify characters on the plates located in the first step. Finally, the information for final processing is stored in an Oracle database. The middleware and the user interface are written in the $\mathrm{C}$ programming language (Fig. 5).

\section{Project results}

Currently, Tehran's quadrilateral prohibited traffic congested area plan (which restricts entry to only a few vehicles every day), is automated and is controlled by over 300 violation registration cameras installed in 104 entry intersections. Before installing the cameras, only 2 out of every 10 violators who entered the prohibited traffic area were fined by police officers, but with the implementation of this system now over $75 \%$ of violators are identified and fined.

Since the recorded images of violators entering the prohibited area has made the detection of plates possible with the help of ANPR technology, all statistical data concerning entry into the prohibited area, regardless of the number of entries, can be processed based on the number of vehicles. Such data can be used for traffic analysis. This can be very valuable to the transportation network and traffic of the city of Tehran where this type of reliable statistical and documented data are not available. For example, statistical data shows that only $16 \%$ of 


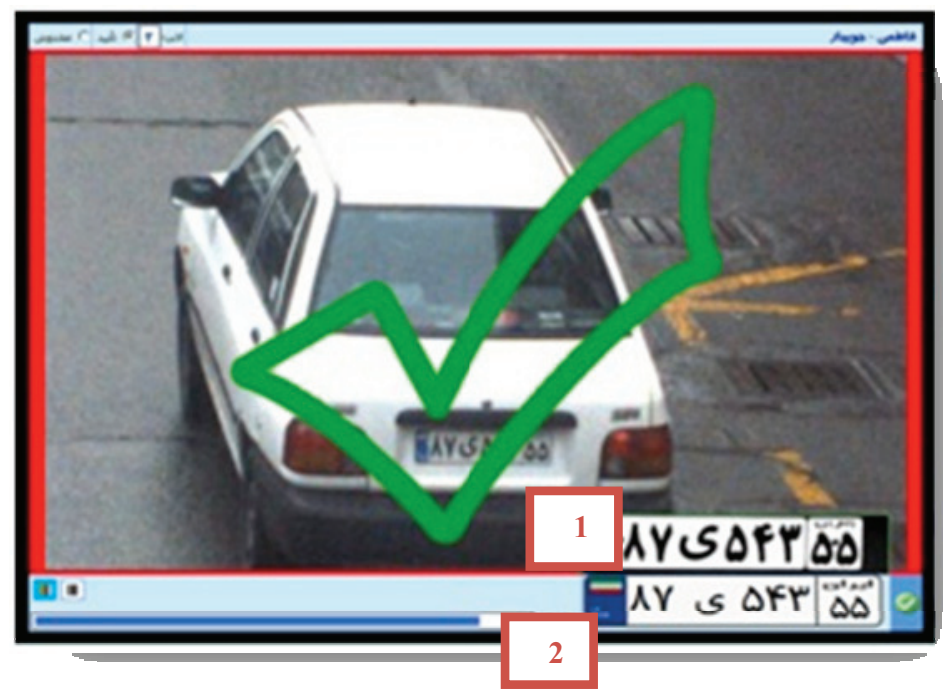

Figure 5: Plate identification software control panel. 1- Image of the plate, read by the ANPR system. 2- Text output by the ANPR system, approved by the operator.

violating vehicles commit one violation per month, and $60 \%$ of delinquent drivers enter the prohibited traffic area more than five times. This, more than anything, shows a pattern of non-urgency of entry in the prohibited area and by identifying these patterns we can find an effective method to reduce these violations. If we take a look at the volume of traffic in entry intersections, there are over 5 million entries into the prohibited area monthly, of which $20 \%$ are unauthorized entries. This shows the scope of this problem and the need for a solution, because even a small reduction in violations involves a large number of vehicles.

As a completely operational result, Figure 6 shows the graphs of the frequency of violations in the 104 intersections mentioned above. The logarithmic curve shows that over $99 \%$ of the violations take place in 85 intersections and by controlling only 10 intersections (less than $10 \%$ of the total), $33 \%$ of all violations are covered. This analysis also showed that $50 \%$ of violations occurred in only 18 intersections which means that if only 18 intersections can be controlled (less than $20 \%$ of the total), $50 \%$ of violations can be stopped. The resulting list of sites with the highest number of violations is the first important achievement of this project, and it has been provided to the police so that they may establish appropriate controls to reduce fraud in these 18 intersections.

The automated control system has other benefits: police officers who have been released from this task can serve other purposes. On the other hand, sometimes drivers try to avoid contact with police officers or create disruptive scenes, and sometimes they argue with police officers in order to avoid getting 


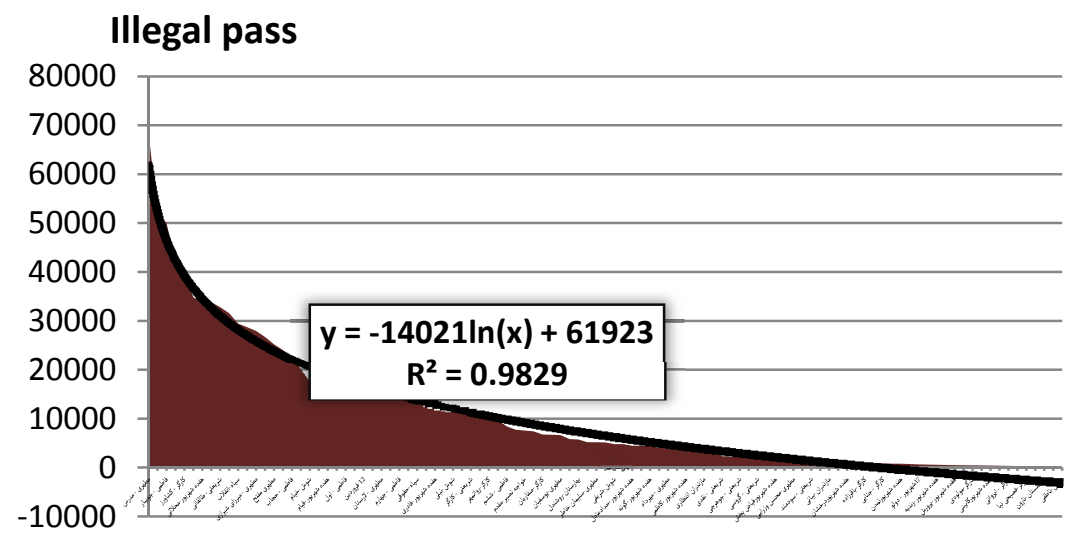

Intersections (Persian names)

Figure 6: Fitted curve to unauthorized traffic data in March 2010.

fined. This is sometimes accompanied by dangerous driving such as entering the prohibited zone in the opposite direction which results in the closing of open lanes. This has been completely eliminated and has led to more effective traffic psychology. Nevertheless, our experience with this technology has also revealed some deficiencies: due to the lack of image-based identifying networks (cameras) inside the prohibited zone and the lack of cameras placed at the exits, it is impossible to track the direction of authorized and unauthorized drivers who cross the prohibited zone in order to detect the goal of their trip. Additionally, traffic studies require basic information about the roadways and their geometrical characteristics such as the number and width of lanes. Also, having traffic statistics for all hours of the day, including the hours that the traffic plan is not in effect, would contribute a great deal to determining trip patterns but this is currently unavailable.

In general, compared to other components of transportation in Tehran, we can consider the automated traffic control system project in Tehran to be a forwardlooking plan whose important achievement in the present circumstances, is to limit unauthorized entry violations in the prohibited area, but whose valuable traffic data must await the upgrade and addition of other informational and statistical components of the transportation network in the future to be fully exploited [11].

\section{References}

[1] Estimated population of cities, the Statistical Center of Iran, 2010. http://www.amar.org.ir/

[2] Comprehensive plan of Tehran in 2008, the Department of Housing and Urban Development http://www.mhud.gov.ir/ 
[3] Database of Tehran (Book I), Tehran Municipality, 2010. http://www.ketabeavval.ir/Tehran/2726.aspx

[4] Wikipedia http://fa.wikipedia.org/

[5] Transport at a glance, the company of Comprehensive studies of transportation and traffic of Tehran (Tehran Municipality), 2007.

[6] Interview with Tehran's roads police chief, Politics Of The Day, November 16, 2010 http://www.siasatrooz.ir/

[7] Interview with the Director of Environmental Protection Agency, Khabar Online, February 8, 2011 http://www.khabaronline.ir/

[8] http://www.cctv-information.co.uk/constant3/anpr.html

[9] Trial Implementation of a congestion tax in Stockholm, 3 of January -31 July 2006, Swedish Road Administration.

[10] "Presentation of operational strategy of automated control of the prohibited traffic area of the city of Tehran."Amin Safai, Dawood Amin Zadeh, Tenth International Conference on Traffic, Tehran, 2010.

[11] Further information available at the project website: www.tehran-congestion-charging.ir 